EGU2020-1508

https://doi.org/10.5194/egusphere-egu2020-1508

EGU General Assembly 2020

(c) Author(s) 2022. This work is distributed under

the Creative Commons Attribution 4.0 License.

\title{
Observations of the Formation of Periodic Plasma Shocks from Fast Mode Waves
}

\author{
Lican Shan ${ }^{1}$, Aimin Du ${ }^{1}$, Bruce Tsurutani ${ }^{2}$, Yasong Ge${ }^{1}$, Quanming Lu $^{3}$, Christian Mazelle ${ }^{4}$, Can \\ Huang ${ }^{1}$, Karl-Heinz Glassmeier ${ }^{5}$, and Pierre Henri ${ }^{6}$ \\ ${ }^{1}$ Institute of Geology and Geophysics, Chinese Academy of Sciences, Beijing, 100029, China (lican.shan.slc@gmail.com) \\ ${ }^{2}$ Pasadena, California, 91109, USA \\ ${ }^{3}$ CAS Key Lab of Geospace Environment, University of Science and Technology of China, Hefei, 230026, China. \\ ${ }^{4}$ Institut de Recherche en Astrophysique et Planétologie, University Paul Sabatier, CNRS, Toulouse, 31400, France \\ ${ }^{5}$ Institut für Geophysik und extraterrestrische Physik, Technische Universität Braunschweig, Mendelssohnstraße 3, 38116 , \\ Braunschweig, Germany \\ ${ }^{6}$ Laboratoire de Physique et Chimie de l'Environnement et de l'Espace, UMR 7328 CNRS, Université d'Orléans, 45100, \\ Orléans, France
}

Collisionless plasma shocks (CPSs), forming when supersonic plasma streams encounter a magnetized obstacle, are invoked to explain the acceleration of ubiquitously energetic cosmic rays. It has long been theorized from magnetohydrodynamics, but not directly observed that the CPSs develop from the growth of small-amplitude, low-frequency plasma waves which excited by reflected ion beams from the obstacle. We present in situ observations of an entire formation sequence of the periodic plasma shocks by the MAVEN spacecraft's magnetic field and particle instruments. The magnetometer first detected small-amplitude circularly polarized magnetosonic waves that further steepened and eventually evolved into periodic shocks. Moreover, differing from the traditional understanding, characterizations of the fast mode waves show that the free energy of the wave/shock generation is provided by newborn protons, and the increasing sunward proton fluxes provided persistent energy for wave steepening. The unusual evidence presents itself from the combination of two circumstances: radial-aligned (Sun-Mars) magnetic fields and Martian atmospheric atom (hydrogen) photoionization and solar wind pickup. These observations lead to the conclusion that newborn ions play a crucial role in the formation process of some CPSs in the astrophysical and space plasma. 